\title{
LA PRECISIÓN GEOMÉTRICAY CONSTRUCTIVA DE LOS SISTEMAS LAMINARES EN LADRILLO Y HORMIGÓN DE LAARQUITECTURA SAGRADA DE SAN JOSÉ DE CÚCUTA
}

\author{
THE GEOMETRIC AND CONSTRUCTIVE \\ PRECISION OF THE LAMINAR SYSTEMS IN \\ BRICK AND CONCRETE OF THE SACRED \\ ARCHITECTURE OF SAN JOSÉ DE CÚCUTA.
}

Yannette Díaz Umaña

Mawency Vergel Ortega ${ }^{2}$

Julio Alfredo Delgado Rojas ${ }^{3}$

Universidad Francisco de Paula Santander.

\section{RESUMEN}

Mediante un estudio de tipo descriptivo se analizan tres sistemas de recubrimiento

$1 \quad$ Magister en Gestión Urbana, Arquitecta, docente y directora del Departamento de Arquitectura, diseño y Urbanismo. Filiación: Universidad Francisco de Paula Santander. Correo electrónico: yannettedu@ufps.edu.co Orcid: https://orcid.org/0000-0003-4582-1593 grupo de investigación Quetelet

2 Doctora en Educación. Postdoctora en Imaginarios y representaciones sociales. Docente y Directora del Departamento de Matemáticas y Estadística. Filiación: Universidad Francisco de Paula Santander. Correo electrónico: mawency@ufps.edu.co. Orcid: https://orcid.org/0000-0001. 8285-2968 grupo de investigación Grautn, Quetelet 3 Magister en Educación Matemática, Arquitecto, Docente. Filiación: Universidad Francisco de Paula Santander. Correo electrónico: julioalfredo@ufps.edu.co Orcid: https://orcid.org/0000-0001-6944-832X grupo de investigación Quetelet en templos icónicos Catedral San José de Cúcuta (1889), iglesia de nuestra Señora de la Candelaria (1952) e iglesia de nuestra Señora del Carmelitas (1969), en Cúcuta-Colombia. Se determinan lasos proyectuales entre la bóveda de crucería y el perfeccionamiento de una red espacial de claves y nervios que apoya sobre ella una delgada cáscara, principio de los sistemas laminares; se determinó el interés de la modernidad en la reducción ornamental y configuración de la estructura vista en la piel del edificio, en una misma solución, explorando los lazos entre forma y estructura, para albergar el uso destinado en la misión litúrgica. Así las 
formas exteriores de envolventes en ladrillo y hormigón, constituyen el contramolde de la envolvente del espacio interior construido por las manos de los mamposteros y fundidores del hormigón que materializaron con precisión formas geométricas simples cargadas de simbolismo.

\section{PALABRAS CLAVE}

arquitectura sagrada, geometría, bóvedas de escrucería, sistemas laminares.

\section{ABSTRACT}

Through a descriptive study, three coating systems are analyzed in the iconic temples in the North-American capital, where influences of medieval architecture for the cathedral of San José de Cúcuta (1889), and concepts of modern architecture of Our Lady of Candelaria (1952) and the Church of Our Lady of the Carmelites (1969). It was known to determine the design lasos between the ribbed vault and the improvement of a spatial network of keys and nerves that supports a thin shell on it, the principle of laminar systems. The interest of modernity in the ornamental reduction and in the configuration of the structure seen in the skin of the building, in the same solution, exploring the links between form and structure, to house the use destined in the liturgical mission. Thus, the exterior forms of these brick and concrete envelopes seem to be the counter-mold of the envelope of the interior space built by the hands of the masons and concrete smelters who precisely materialized simple geometric shapes loaded with symbolism.

\section{KEYWORDS}

sacred architecture, geometry, scruffy vaults, laminar systems.

\section{INTRODUCCIÓN}

Con el propósito de investigación de los sistemas laminares en ladrillo y hormigón de la arquitectura sagrada de san José de Cúcuta, fue necesario identificar en la muestra de población a estudiar, tres estructuras cuyo punto de partida constructiva se dan hacia 1889, 1952 y 1969 con la catedral de San José de Cúcuta, Iglesia de Nuestra Señora de la Candelaria y la iglesia de nuestra señora del Carmelita respectivamente, cuyas materializaciones arquitectónicas se relacionan con influencias de importantes momentos históricos como el medieval y moderno.

\section{METODOLOGÍA}

Mediante un estudio de tipo descriptivo se analizan tres sistemas de recubrimiento en los templos icónicos en la capital nortesantanderana, donde se determinan influencias de la arquitectura medieval para la catedral San José de Cúcuta (1889), y conceptos de la arquitectura moderna para la iglesia de nuestra Señora de la Candelaria (1952) y la iglesia de nuestra Señora del Carmelitas (1969). Se realiza el análisis desde el impacto estilístico, geométrico y constructivo, visto en estas obras icónicas, la primera dada por ser el antecedente histórico de este tipo de sistemas laminares, la segunda por ser pionera a nivel nacional y la tercera por constituirse en uno de esos pocos casos a nivel mundial de esta tipología laminar de tipo prismático semiradial. Se plantea inicialmente el ámbito urbano, al considerar las edificaciones consagradas para la misión litúrgica y la administración de los sacramentos, un lugar necesario para el encuentro, de manera contextualizada y vinculadas a procesos sociales y políticos, que han figurado su papel simbólico, pedagógico, expresivo y fenomenológico (Díaz, Vergel \& Delgado 2020). Por ejemplo, la Catedral de san José constituye elemento jerárquico dentro del paisaje arquitectónico de la ciudad (Vergel, Díaz \& Delgado 2020). Así este edificio sagrado adopto esquemas representativos entre las vertientes gótica, neoclásica, románica, y ecléctica; 
acogidos desde el orden gubernamental, órdenes religiosas y burguesía (Díaz, Delgado \& Vergel 2021). Estas interacciones explican la amplitud e inexactitud del término republicano en la arquitectura del siglo XIX y principios del XX (Vergel, Delgado \& Díaz 2018).

De la misma forma Díaz, Delgado \& Vergel (2021) proponen estimar algunas características urbanas relacionadas con las morfologías constructivas:

"En consecuencia, esta dimensión estilística, regulada con la condición temporal y la espacial, cuanto más se acerca a la plaza principal es dominada por influencias neoclásicas como en el Capitolio Nacional y en su lejanía distingue nuevos modelos arquitectónicos, más cercanos a la modernidad." ( $p$. 382)

En el caso de la ciudad de Cúcuta, la zona cercana al parque Santander se consolido con una arquitectura ecléctica con las tradicionales viviendas y edificios gubernamentales levantados a finales del siglo XIX. De ahí que la catedral de San José de Cúcuta presente una variedad de estilos arquitectónicos tomados del extranjero como solía suceder con la arquitectura colombiana en la época de la república. Así las condiciones espacio temporales, de la catedral, el proceso constructivo lento que dura más de 80 años, ya que la primera piedra se remonta al 1889, y su última transformación notable de la estética arquitectónica fue en 1969, proceso en el cual se adoptan varios estilos medievales, distinguibles en el marco arquitectónico del Parque Santander, el núcleo principal de la ciudad.

Para la iglesia de nuestra Señora de la Candelaria estas condiciones espacio-temporales de su construcción varían, pues se llevó a cabo hacia 1952 a 25 kilómetros de la plaza principal, y su arquitectura fue proyectada con un estilo moderno, prácticamente esta construcción se distingue como pionera en Colombia. A su vez la iglesia de nuestra señora la candelaria a 7 kilómetros de la plaza principal y construida en 1969 se proyectó con un estilo de arquitectura propia de la última década de la modernidad. (figura 1)

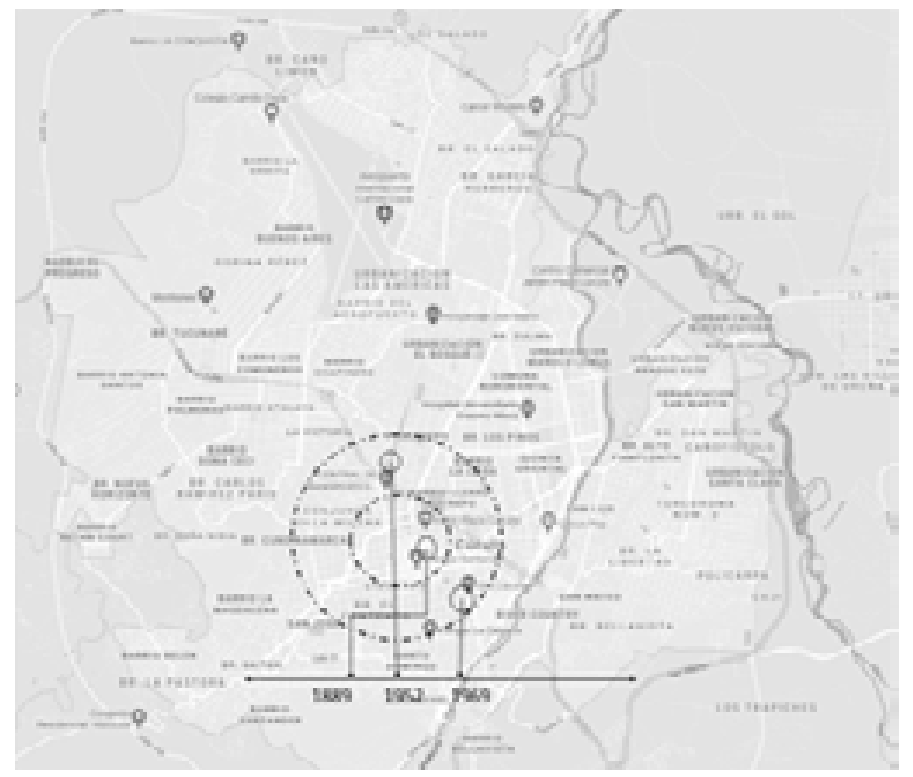

Figura 1. Ubicación espacio temporal de los templos a estudiar en el plano de la ciudad de Cúcuta. Fuente. Elaboración propia con plano de https://www.google.com/maps/ 
De acuerdo a esto, el origen y desarrollo de nuevas formas estructurales y arquitectónicas en los siglo $\mathrm{XIX}$ y principios del $\mathrm{XX}$, estuvo íntimamente ligado al extraordinario desarrollo constructivo alcanzado al momento, que propicio un desarrollo sin precedentes de los elementos curvos suspendidos como la cúpula y las bóvedas de la arquitectura sagrada, dando posteriormente con la madurez tecnológica en la concepción estructural, avance de las propiedades de los materiales estructurales conocidos, así como técnicas para propiciar menor peso, mayor economía, propias de la modernidad, el origen a las formas laminares curvadas como estos casos de iglesia de Nuestra Señora de la Candelaria y estructuras plegadas en la iglesia de nuestra Señora del Carmelitas. En consecuencia, las amplias posibilidades tecnológicas en la arquitectura, favorecieron un proceso de evolución morfológico de la estética arquitectónica clásica tradicional de los templos con una sabiduría constructiva a una arquitectura más atrevida consiente de explorar formas livianas expresivas y resistentes.

\section{ANÁLISIS Y DISCUSIÓN}

El orden constructivo de la Catedral de San José de Cúcuta, constituye el empeño de los habitantes por la consagración del espacio urbano. Para ello la imagen perfecta del templo, debía reinterpretar los referentes internacionales de arquitectura sagrada, confiriendo una estética cosmopolita acorde a la apertura económica de la ciudad de san José de Cúcuta (Díaz, Delgado \& Vergel 2020). De este modo la reinterpretación de estilos medievales, no tenía otra razón que sorprender, cautivar y conmover al feligrés. Este objetivo fenomenológico es claro, y posteriormente se daría en continuidad en la arquitectura sagrada moderna, definiendo sistemas innovadores que no pasan desapercibidos, como ocurre en las iglesias Señora de la Candelaria y nuestra Señora del Carmelitas. En especial en lo que se refiere a la catedral de san José de Cúcuta Delgado, Díaz \& Vergel (2021) afirman:

"visto en lo experimental y contradictorio de las formas arquitectónicas, haciendo alusión al repertorio que va desde la edad media hasta el 2 imperio donde se destaca el neoclásico, neorromántico, neogótico entre otros movimientos italianizantes y franceses, por lo que la arquitectura exterior, su decoración de interiores con sus particulares mezclas agregaban suntuosidad, complejidad, pero sobre todo mundo." (p. 215)

De este modo, la catedral de san jose de Cucuta, se organiza en un esquema de cruz latina con un sistema escalonado de naves (Vergel, Delgado \& Díaz 2020), donde el sistema laminar puede reflejarse como antecedente historico en las bovedas de escruceria. Asi la bovedas estudiadas cubren las naves laterales con un area de $663.54 \mathrm{~m} 2$ y la nave central con un area $513.23 \mathrm{~m}^{2}$ lo que corresponde a una superficie $48 \%$ del area total del templo. La conformación de las bóvedas de escrucería y la misma cúpula en la catedral de san José, adquieren un lenguaje estilístico ecléctico (Delgado, Díaz \& Vergel (2019) y expresivo que sorprende al visitante (Vergel, Díaz \& Delgado 2020). Estas estructuras similares a las láminas curvadas están constituidas por figuras geométricas simples distinguidos en las porciones de cilindros o esferas, luciendo su construcción nervada para formar la bóveda (imagen 2). Zaragoza, (2008) la define como:

"Podemos considerar una bóveda de crucería como aquella en la cual la forma de la bóveda se logra mediante el cruce de arcos diagonales llamados también nervios. Estos arcos sirven de directriz para el trazado de unas superficies de doble curvatura que llamamos plementerías". (p. 99). 
Aun cuando específica, mencionando a Jacques

Heiman (1995) que:

“...el nervio cumple una función estructural muy necesaria como refuerzo de las aristas, aunque puede no ser esencial. Además, como se ha visto, facilita la

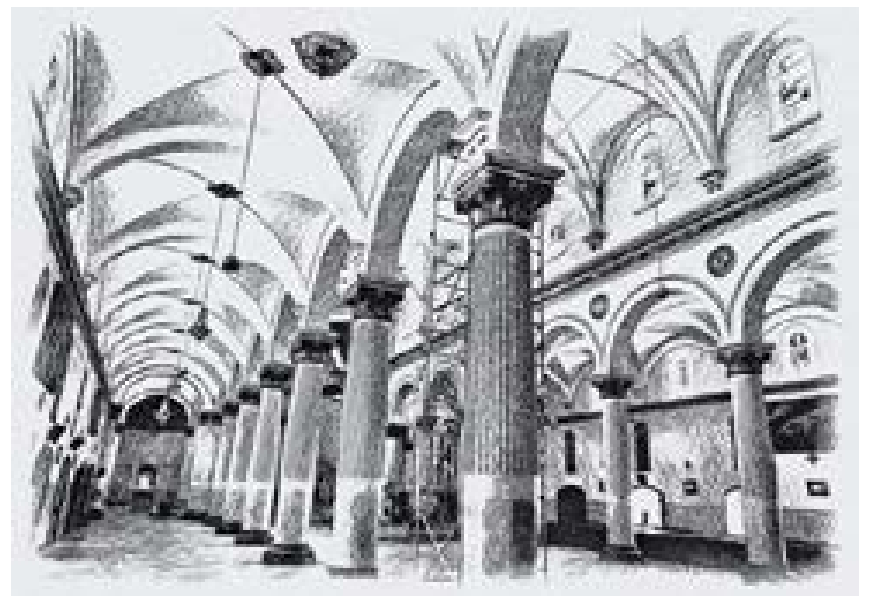

construcción de los plementos, permite eliminar parte de las obras auxiliares y cubre las juntas defectuosas de las aristas. Finalmente, el nervio puede desempeñar un papel estético." (Zaragoza, 2008, p. 105).

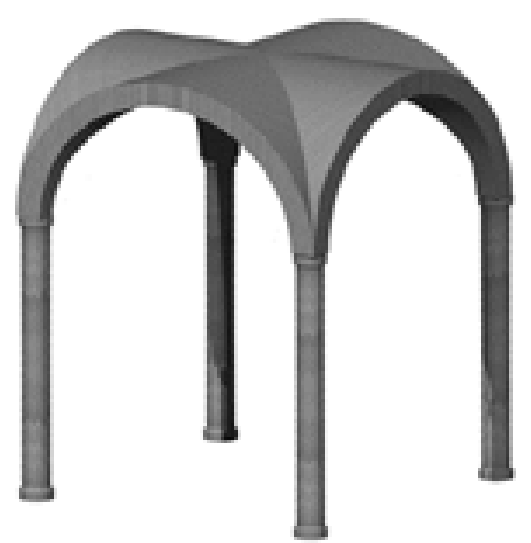

Figura 2. Perspectiva interna vista en primer plano las bóvedas de escrucería en la nave lateral izquierda de la catedral y modelo tridimensional de unidad básica Fuente: Autores

Por consiguiente, en la Catedral de San José, las nervaduras tienen una geometría que se ajusta a su sistema constructivo y se refleja en el despiece de los elementos que las forman, al igual que la arquitectura moderna asumiría como precepto fundamental la abstracción matemática (Díaz, Delgado \& Vergel 2021). En referencia a la bóveda, existen innumerables variantes, desde el cruce de dos cilindros hasta la bóveda por arista de cuatro semi esferas, que en general siempre utilizan superficies regladas radiales (conos y cilindros) o esferas, como en este caso particular que representa el cielo.

En efecto la bóveda de crucería cuadripartida de la catedral, de planta cuadrada, está estructurada con arcos de medio punto rebajado, los cuales determinan la altura de la clave central (nodo superior o cruce de arcos), de allí la inspiración se halla en diversas corrientes hasta ubicarse en la denominación ecléctica (Delgado, Díaz \& Vergel 2019), por cuanto es un estilo arquitectónico de los siglos XIX y XX en el que una sola obra incorpora una mezcla de elementos de estilos históricos anteriores, (góticos, bizantinos, clásicos, románico). El número de arcos observados en cada unidad son 6 , que se justifican constructivamente como intento de eliminar las cimbras necesarias para construir las plementerías.

Toda la unidad construida en ladrillo, con alta técnica de mampostería, permite con exactitud la precisión de arcos y plementos. Así los nervios resuelven la construcción de la plementería en apoyos cómodos, facilitan el trabajo constructivo y permiten visualizar la conformación de geometrías básicas implementadas. Por ello, se considera la red de crucerías como una cimbra perdida ejecutada en ladrillo para este caso constructivo. Dicho de otra manera, por Fortea (2015) quien declara que la construcción de superficies abovedadas se hace gracias al procedimiento de inclinar las 
hiladas, en el aire sin medios auxiliares. Por su parte, Huerta (2004) declara que la bóveda de crucería experimenta notable desarrollo en la historia, no solo por su complejo diseño de crucerías en el plano horizontal, sino por el perfeccionamiento de una red espacial de claves y nervios que puede adoptar la forma más oportuna para apoyar sobre ella una delgada cáscara: la plementería. Así "su geometría se basa en el equilibrio de las fuerzas estáticas y estructurales. Esta combinación entre fuerza y movimiento- la estructura mecánica- definen la forma arquitectónica." (Fortea 2015, p. 34).

En segundo lugar, antes de hacer referencia a la iglesia de la candelaria y su momento constructivo en la modernidad y en un contexto urbano retirado del centro de la ciudad, es necesario resaltar la racionalización de las formas con el empleo de sistemas matemáticos en la arquitectura moderna, que facilitó la libertad estilística definiendo configuraciones y geometrías igualmente sorprendentes y atractivas (Díaz, Díaz, \& Galvis 2021) como las derivadas del historicismo. A cambio se percibe los efectos de simplificación simbólica de las iglesias en el Siglo XX, algunas materializadas tan solo por una membrana con perfil curvo que les concedía la propiedad de mantener las cualidades de religiosidad artísticas del edificio sagrado manteniendo el misticismo de los antiguos templos (Díaz, Delgado \& Vergel 2021). Se explica de esta manera el sistema laminar de hormigón en perfil curvo, de la iglesia de nuestra Señora de la Candelaria, modelo representativo, que han sido motivo de estudio en otros contextos, constituyendo una importante contribución a la memoria Nacional (Díaz, Delgado \& Vergel 2021). En ella se advierte tres elementos de orden progresivo, muy probablemente correspondiendo al concepto escalonado de las tres naves propias de la arquitectura medieval, pero transformando notoriamente su morfología. Estas tres superficies actúan como la cubierta del templo de Nuestra Señora la Candelaria, integradas en un solo gesto plástico, sobre su planta rectangular de $721 \mathrm{~m} 2$ y sin apoyos internos. (imagen3).
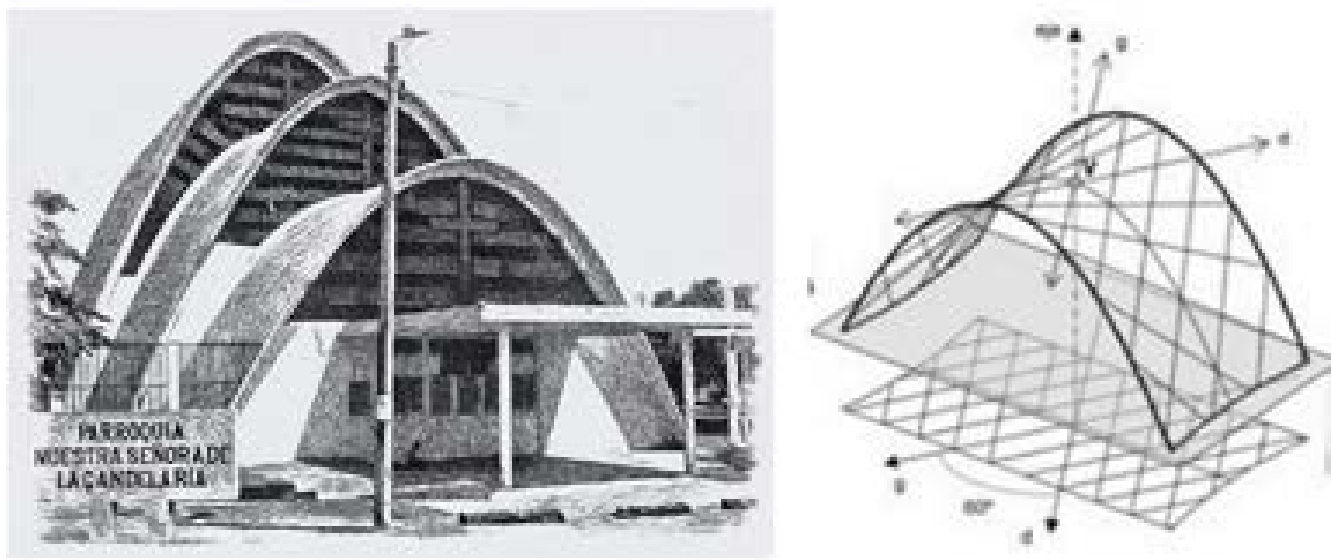

Figura 3. Perspectiva Iglesia de nuestra señora de la candelaria con Modelos en $3 \mathrm{~d}$ del sistema laminar. Fuente: Elaboración propia

La organización en el interior está encauzada a una sola nave, por lo que la desenvoltura de la superficie la hace particular con sus tres estructuras laminares de perfil catenario de elevación progresiva. Las tres secciones corresponden a una de menor dimensión (18m. de luzy $10 \mathrm{~m}$. de altura), mediana dimensión ( $20 \mathrm{~m}$. de luz y $15 \mathrm{~m}$. de altura), y la de mayor dimensión (22m. de luz y $20 \mathrm{~m}$. de altura). El sistema logra disminuir el espesor de la lámina con la ayuda del hormigón y el entramado de viguetillas de $10 \mathrm{~cm}$ de sección, cada una con cuatro varillas 
redondas de acero de 1/4" (Galindo, Salazar, y Henao, 2018), que conforman su piel curva de carácter auto portante. Lo cual permite analizar la diminución radical de la sección constructiva de $20 \mathrm{~cm}$ de las bóvedas de escrucería de la catedral con referencia a la sección de $10 \mathrm{~cm}$ de superficie ligera de hormigón de la cubierta de la iglesia la candelaria con forma catenaria:

"En matemáticas y arquitectura se recurre a la palabra catenaria para distinguir la curva que sigue la forma que adquiere una cadena o cuerda de densidad uniforme y perfectamente flexible sujeta por sus dos extremos y que se encuentra sometida únicamente a las fuerzas de la gravedad (de Zárraga (2003). Esta forma es usada en la arquitectura e ingeniería muy resistente a la tracción y compresión en respuesta a las fuerzas generadas por la gravedad."(Díaz, Delgado, \& Vergel, 2021, p.20)

La morfología de la iglesia de Nuestra señora de la Candelaria es una curva catenaria invertida. Es decir, matemáticamente las características de la catenaria se conservan al invertir su gráfica (imagen4). Para Huerta (2004) por su parte:
"En los arcos de formas catenarias o cuasi-catenarias son consideraciones, y no el espesor infinitesimal del arco límite, las que deciden el proyecto. La esbeltez límite se reduce a gran velocidad a medida que el arco se va haciendo más rebajado, y todos los arcos con una flecha inferior, digamos, a $1 / 4 \mathrm{de}$ la luz son prácticamente catenarios, con espesores límite inferiores a $1 / 140$ de la luz, llegando a 1/4.000 para una flecha (no infrecuente en las bóvedas de forjado) de 1/10 de la luz." (p. 395)

De modo que el arco catenario es la forma ideal para sobrellevar su propio peso. Ostenta la ventaja que cuando mayor es la altura, más pequeño es el esfuerzo horizontal en los puntos de arranque, con lo que se pueden obtener grandes alturas con mínimos empujes laterales. Se explica con esto que la estructura más alta tiene mayor resistencia en sus laterales. Se distinguen otros elementos estructurales de $84 \mathrm{~cm}$ y $28 \mathrm{~cm}$, que le confieren la estabilidad, al perfil dando lugar a los 6000 ladrillos de arcilla de $23 \mathrm{~cm}$ de ancho y $34 \mathrm{~cm}$ de largo, que se comportan como una unidad permitiendo alcanzar el espacio abierto del auditorio sin columnas, respondiendo a la libertad espacial propuesta en la modernidad.
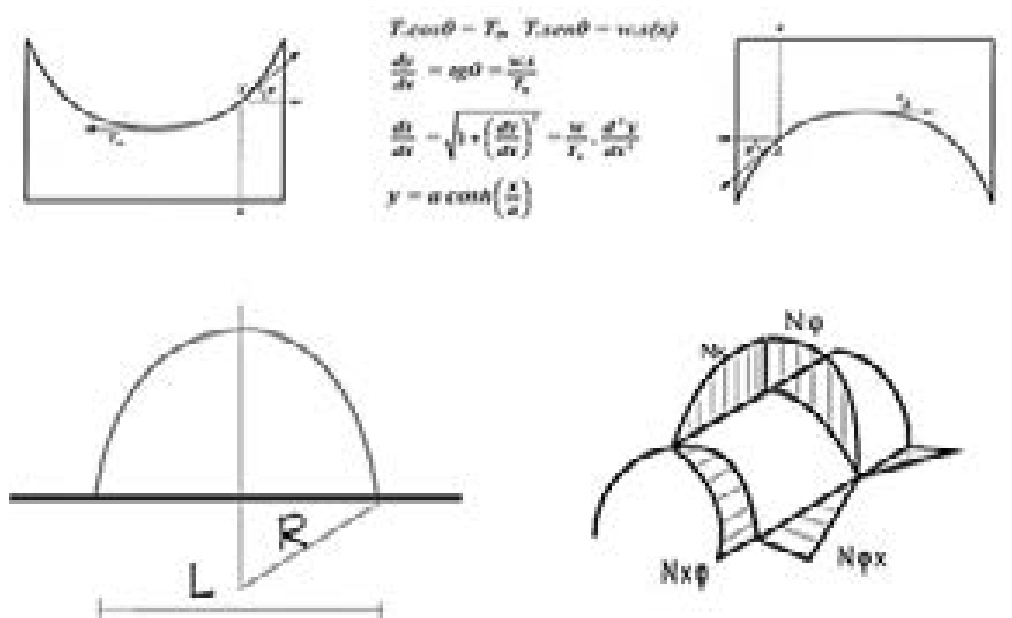

Figura 4. Forma catenaria del sistema laminar de la iglesia de nuestra Señora de la Candelaria. Fuente: Autores 
En general se observa, de estas dos estructuras (bóvedas de crucería de la catedral de San José de Cúcuta y la correspondiente al sistema laminar en forma catenaria de iglesia de Nuestra señora de la Candelaria descrita por Díaz, Delgado \& Vergel (2021), que comparten el uso del ladrillo como manufactura lograda por la materia prima de la región arcillera. Además, tanto para las bóvedas de escrucería de la catedral como para el sistema laminar en forma catenaria de la iglesia de nuestra señora de la Candelaria son las fuerzas de tracción que padecen los arcos, que se transmite a través de la propia curva hacia los apoyos. En el caso de la catedral los esfuerzos se distribuyen a su vez en muros y las 16 columnas de la nave central y en el caso de la Candelaria los esfuerzos los reciben solo y directamente a los cimientos. Es esta propiedad, especialmente hablando del arco catenario hace que no necesite apoyo lateral para sustentarse.

Para Díaz, Delgado \& Vergel (2021) todo este sistema de viguetillas y ladrillos queda mimetizada en el enlucido de mortero favorable para la impermeabilización, aplicado en la cara externa de la membrana curvada, que la hace aparentar ser una estructura totalmente en hormigón, y de esta manera evidenciar la coherencia con los ideales modernos. De acuerdo a esta caracterización, el modernismo en ciudades como San José de Cúcuta, se percibe como una secuela del eclecticismo, pues se configura como un estilo, en donde en una sola obra, se aportan varios conceptos y variedad de materiales vernáculos, manufacturados de forma artesanal, e industrializados, conforme a referentes europeos (Vergel, Delgado \& Díaz 2020).

En tercer lugar, en la iglesia de nuestra Señora del Carmelitas ubicada temporalmente a finales de la modernidad, periodo definido hacia los últimos años la década de los 60 (Díaz, Díaz y Galvis 2021), cuando se consolida la idea de la estructura optimizada en el funcionamiento y en la naturaleza de sus esfuerzos, permite crear formas más libres y afirmadas en geometrías estrictas. Con esta concepción Díaz, Delgado \& Vergel (2021) definen las formas laminares en hormigón, como la técnica estructural de plegaduras, que conforman sistemas tridimensionales con la unión de sus aristas en ángulos, de modo que las delgadas láminas de hormigón armado se caracterizan por sus perfiles planos articulados con bordes pronunciados que le dan la rigidez. Este sistema se da en oposición al de las láminas curvas cuya característica está dada por su arqueamiento simple o de doble curvatura. Pero de igual manera, tanto para la arquitectura sagrada con influencia medieval y la arquitectura moderna vista en estos tres modelos, los sistemas laminares curvas o laminares plegadas revelan propiedades plásticas y constructivas, haciendo notoria la jerarquía ascendente hacia el presbítero, mostrada en la direccionalidad al altar con las formas arquitectónicas. De menor a mayor grado se le reconoce su propiedad casi ingrávida, al dotar al espacio de las naves del plasticismo propio de la arquitectura sagrada con sentido simbólico. Con esta perspectiva se reconocen en estos cuerpos abovedados o plegados un significado cosmológico y misterioso, en el sentido artístico y constructivo (Figura 5). Dada esta ilustración, la superficie que protege la iglesia de Nuestra Señora del Carmen corresponde a un sistema laminar plano articulado por arista o plegada, con una luz libre de $35 \mathrm{~m}$., cuyas líneas de intersección forman las correspondientes limatesas y limahoyas, formando 14 secciones triangulares que cubren un área total de $1200 \mathrm{~m} 2$. Se trata, por tanto, de un tipo estructural que permite salvar grandes luces pese a su escaso espesor de $8 \mathrm{~cm}$. 

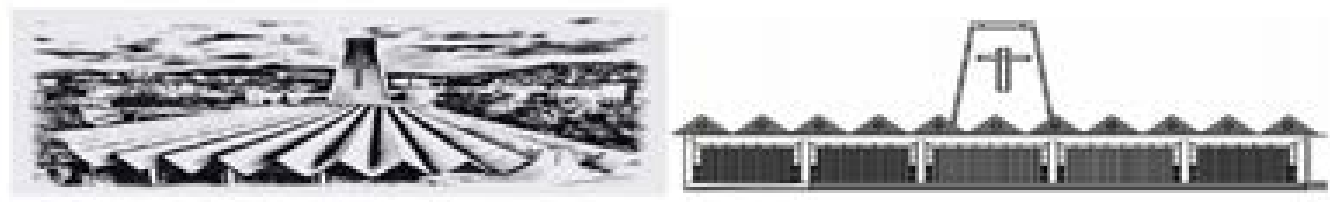

Figura 5. Forma del sistema laminar plegado de la iglesia de nuestra Señora del Carmelitas. Fuente: elaboración propia con levantamiento de fachada frontal perfilada por: Gabriela Aguirre, Marvin Pérez, Jesús Álvarez.

El rigor transversal gracias a su forma de plegadura, principalmente a la arista que se forma en el pliegue, presta oposición a los esfuerzos, siendo los planos que la forman los que impiden que esta se pandee según Baldanta (2015) en Díaz, Delgado \& Vergel (2021).

"estas plegaduras delgadas presentan menores requerimientos con los trabajos del encofrado (García, 2.013) referido a superficie en hormigón curva. para ambos tipos de estructura, el control y calidad en sus técnicas de elaboración, es exhaustiva Coca (2.014), además requiere formaletas $y$ andamiajes con cantidad precisa, por lo cual mano de obra debe ser capaz en número y habilidad para construcción de estas formas laminares." (Díaz, Delgado \& Vergel 2021, p 9)
De esta manera el comportamiento longitudinal de estas losas, son como las de una viga apoyada en los extremos, a su vez, transversalmente, funciona como una viga continúa apoyada en los pliegues, con menor rigidez. Otra propiedad es la comentada por Angerer en García (2.013) es la comparativa facilidad con menores requerimientos en los trabajos del encofrado, en relación a las de superficie en hormigón curva. No obstante, la técnica de elaboración para ambas superficies, requiere formaletas y andamiajes especiales con una mano de obra capaz de precisar los detalles para su construcción. En especial esta plegadura en hormigón con formas estructurales lineales que se conciben paralelamente unas a otras en forma de abanico. En este sentido Díaz, Delgado \& Vergel (2021) clasifica la iglesia de Nuestra Señora del Carmen, al sistema laminar semiradial, ya que su origen se da en puntos muy próximos que consolidan esa direccionalidad en forma de abanico. De este modo, la envolvente se apoya sobre sobre el altar debajo de la torre y sobre el pórtico de la entrada principal. Su longitud sobrepasa la fachada frontal, dejando ver el voladizo sobre el atrio, que forma parte del atractivo estético y funcional de este tipo de envolventes. (Figura 6). 


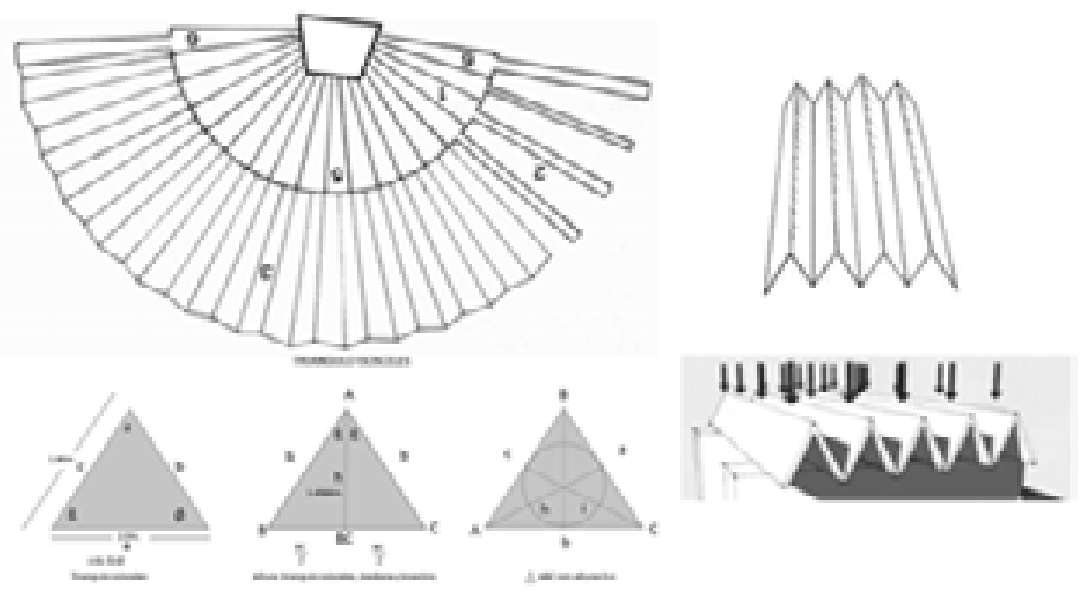

Figura 6. Forma del sistema laminar plegado semiradial de la iglesia de nuestra Señora del Carmelitas. Fuente: elaboración propia

En particular las losas de concreto conforman una geometría regular, basada en la forma simple de la plegadura, con sistema de láminas que posibilita la cubierta de la iglesia de Nuestra Señora del Carmen, donde el rigor geométrico, se logra con la integración entre elementos y por las fuerzas de cohesión, de modo que las fuerzas internas se distribuyen a lo largo de sus bordes, dando resistencia a todo el elemento. Esta sincronía de agrupaciones geométricas correspondientes al triangulo, como representación de la Trinidad, con una medida de $2 \mathrm{~m}$ en cada uno de sus lados y $1.8 \mathrm{~m}$ la base, de cada triangulo isósceles que viabilizan el funcionamiento de la estructura. De esta manera aun cuando la arquitectura moderna racionaliza conceptos proyectuales, da continuidad a la importancia del espacio místico de los templos, alcanzado con la cubierta en plegadura de tipo prismático semiradial en este ejemplo de la ciudad de Cúcuta.

\section{CONCLUSIÓN}

La definición y configuración de estos sistemas laminares se logra incorporando en al proceso proyectual órdenes matemáticos y geométricos que le permiten consolidar un espacio fenomenológico al templo. En la catedral el espacio místico y alegórico se puede hallar en la proyección de bóvedas de crucería cuadripartita suspendidas sobre la nave central y laterales, cuyos apoyos en los muros y en las 16 columnas centrales dejan al descubierto el carácter artístico, tecnológico, pero sobre todo conmovedor para el feligrés que encuentra en esta superficie un éxtasis sobrecogedor al ingresar al templo.

La iglesia de nuestra Señora de la Candelaria con el sistema laminar en forma catenaria, no solo resuelve la morfología cambiando el paradigma estilístico, con la misma fuerza expresiva de la arquitectura sagrada tradicional, generando un skyline especial por su libertad plástica sobre la ciudad de Cúcuta. Al interior consigue conmover también al visitante, así el misticismo es logrado con las tres láminas curvas progresivas en altura suspendidas sin apoyos intermedios.

Con la iglesia de nuestra señora del Carmelitas, queda claro que el interés de la modernidad además de la reducción ornamental, era un notable provecho por la configuración de la estructura vista en la piel del edificio, en una misma solución. Así las estructuras contenedoras de estas dos iglesias exploran la relación entre forma y estructura, garantizando la unificación entre el espacio interior y el volumen exterior, a la vez que define un nuevo modelo arquitectónico que plantea la estructura 
como un contenedor fenomenológico capaz de albergar el uso destinado a la misión litúrgica.

De este modo se observó para los tres sistemas vistos en la catedral de San José de Cúcuta, la iglesia de nuestra Señora de la Candelaria y la iglesia de nuestra Señora del Carmelitas, que las formas exteriores de estos envolventes en ladrillo y hormigón, parecen ser el contra molde de la envolvente del espacio interior construido magistralmente por las manos de los mamposteros y fundidores del hormigón. $Y$ es la forma de ese espacio interior, muy singular con el valor suficiente para conmover al feligrés y adentrarlo en el espacio consagrado constituido por figuras geométricas simples cargadas de simbolismo.

\section{REFERENCIAS BIBLIOGRÁFICAS}

Delgado Rojas, J. A.., Díaz Umaña, Y.., \& Vergel Ortega, M.. (2019). El paisaje Arquitectónico y sonoro del campanario de la Catedral de San José de Cúcuta. Revista Logos, Ciencia \& Tecnología, Vol. 11, No. 1.

Delgado Rojas, J. A.., Díaz Umaña, Y.., \& Vergel Ortega, M. (2021). Valoración patrimonial a partir del análisis morfológico y matemático de San Pedro y San Pablo, de la catedral de San José de Cúcuta. Revista Boletín Redipe, 10(7)

Díaz Umaña, Y.., Delgado Rojas, J. A.., \& Vergel Ortega, M. (2021). Representaciones socioespaciales, en la memoria histórica de la catedral y su entorno urbano en San José de Cúcuta. Revista Boletín Redipe, 10(4).
Díaz Umaña, Y.., Delgado Rojas, J. A., \& Vergel Ortega, M. (2020). La geometría constructiva del cimborrio de la catedral de San José de Cúcuta. Revista Boletín Redipe, 9(12), 294-299. https://doi. org/10.36260/rbr.v9i12.1156

Díaz-Umaña, Y.., Vergel-Ortega, M \& DelgadoRojas, J. A. (2020). Modelo geométrico y arquitectónico de la cúpula mayor en San José de Cúcuta. Revista Boletín Redipe, 9(3), 160-166. https://doi. org/10.36260/rbr.v9i3.941

Díaz-Umaña, Y., Delgado-Rojas, J. A.., \& Vergel-Ortega, M. (2021). La geometría basada en la malla como mecanismo compositivo de Villa Savoye. Eco Matemático, 12(1). https://doi. org/10.22463/17948231.3070

Díaz-Umaña, Y.., Delgado-Rojas, J. A.., \& Vergel-Ortega, M. (2021). Una tendencia de la arquitectura moderna en América Latina, basada en la Geometría revolucionaria de la iglesia de San Francisco de Asís. Eco Matemático, 12(2).

Díaz-Umaña, Y., Díaz- Fuentes, X. \& GalvisCenturión, R. (2021). 35 proyectos de arquitectura contemporánea. Materia forma \& contexto. ECOE ediciones. Bogotá.

Díaz, Y., Delgado, J. \& Vergel, M. (2021). Estructura como envolvente en la iglesia nuestra Señora del Carmen. Revista Boletín Redipe vol. 10 no. 8

Díaz, Y., Delgado, J. \& Vergel, M. (2021). Geometrías estructurales aplicadas a la arquitectura de la iglesia de nuestra Señora de la Candelaria. Revista Boletín Redipe vol. 10 no. 8 
Galindo Díaz, Jorge, Salazar Marulanda Carolina, Henao Laura. (2018). Cubiertas laminares en cerámica armada: los aportes del ingeniero Guillermo González Zuleta (Colombia, 1947-1962). (Informes de la Construcción, 70,551, (2018): 1-12. Consulta en noviembre 26 2019: https:// doi.org/10.3989/ic.60713

Huerta, Santiago (2004). Arcos, bóvedas y cúpulas. Geometría y equilibrio en el cálculo tradicional de estructuras de fábrica. Instituto Juan de Herrera, Escuela técnica superior de arquitectura. Madrid

Fortea, Teresa (2015). Desmaterización de la envolvente estructural Contemporánea. Una secuencia de estrategias arquitectónicas en Toyo Ito, Herzog \& de Merau y Sanaa. Tesis doctoral. Universidad Politécnica de Madrid.

Vergel-Ortega, M; Delgado Rojas, J \& Díaz Umaña, Y (2018). Cathedral bell's San José de Cúcuta: Heritage and acoustics. Journal of Physics: Conference Series, Volume 1329, XIV Applied Mathematics Meeting and XI Statistics Meeting 7-8 December 2018, San José de Cucuta, Colombia

Vergel Ortega, M., Díaz Umaña, Y.., \& Delgado Rojas, J. A. (2020). Luz, símbolo y revelaciones a través del papiro de Jeremías y la presencia de la virgen María en la catedral de San José de Cúcuta. Revista Boletín Redipe, 9(11), 272-281.
Vergel Ortega, M.., Delgado Rojas, J. A.., \& Díaz Umaña, Y. (2020). Estudio iconográfico y geométrico del vitral de San José en la catedral de Cúcuta. Revista Boletín Redipe, 9(6), 119-133. https://doi. org/10.36260/rbr.v9i6.1006

Vergel Ortega, M.., Delgado Rojas, J. A.., \& Díaz Umaña, Y. (2020). Dimensión histórica de la red ferroviaria, su impacto económico y urbano en San José de Cúcuta. Revista Boletín Redipe, 9(6), 183-191. https://doi.org/10.36260/rbr. v9i6.1011

Vergel Ortega, M.., Díaz Umaña, Y. \& Delgado Rojas, J. A (2021). Dependencia geométrica en la composición del vitral del Espíritu Santo. un estudio iconográfico y arquitectónico en catedral de san José de Cúcuta. Revista Boletín Redipe (10). 8

Zaragoza, Arturo (2008). A propósito de las bóvedas de crucería y otras bóvedas medievales. Anales de Historia del Arte 2008, Volumen Extraordinario 99-126. \} 